\title{
INDICADORES DE TRATA DE PERSONAS EN MUJERES QUE EJERCEN LA PROSTITUCIÓN EN LOCALES DE ALTERNE DE LA COMUNIDAD DE MADRID (ESPAÑA)
}

\author{
INDICATORS OF TRAFFICKING IN WOMEN \\ PRACTICING PROSTITUTION IN STRIP \\ CLUBS IN THE COMMUNITY OF MADRID \\ (SPAIN)
}

\author{
Eva A. SILVA ${ }^{1}$, ANTONIO L. MANZANERO ${ }^{2}$, \\ GARAZI BENGOA ${ }^{3}$ Y MARÍA JOSÉ CONTRERAS ${ }^{4}$
}

Cómo referenciar este artículo/How to reference this article:

Silva, E. A., Manzanero, A. L., Bengoa, G. y Contreras, M. J. (2018). Indicadores de trata de personas en mujeres que ejercen la prostitución en locales de alterne de la Comunidad de Madrid (España) [Indicators of trafficking in Women Practicing Prostitution in strip clubs in the Community of Madrid (Spain)]. Acción Psicológica, 15(1), 1-16. https://doi.org/10.5944/ap.15.1.19864

\section{Resumen}

El objetivo del trabajo fue analizar la situación de las mujeres que ejercen la prostitución en el entorno de la Comunidad de Madrid (España), en el contexto de locales de alterne y la posibilidad de encontrar víctimas de redes de trata de personas. Se analizaron entrevistas a 180 mujeres que ejercían la prostitución en locales de la periferia de Madrid. Los resultados mostraron que la mujer que se dedica a la prostitución tiene una media de 32.57 años; proceden de Latinoamérica, Europa y en menor número de África; en su mayoría tienen algún tipo de estudio. Un $80.5 \%$ dijo tener familia, y un $68 \%$ tenían entre 1 y 4 hijos. El análisis de las preguntas respondidas o evitadas, permitió concluir que omiten información relevante en preguntas sobre los indicadores de trata y explotación, lo que hace sospechar que pudieran estar instruidas para no facilitar información sensible,

Correspondencia: María José Contreras. Departamento de Psicología Básica I. Universidad Naional de Educación a Distancia (UNED).

Email: mjcontreras@psi.uned.es

ORCID: Eva A. Silva (https://orcid.org/0000-0002-5403-9766), Antonio L. Manzanero (https://orcid.org/0000-00032619-4571), Garazi Bengoa (https://orcid.org/0000-0002-9502-5387) y María José Contreras (https://orcid.org/00000001-6302-5238).

${ }^{1}$ Guardia Civil, España.

${ }^{2}$ Universidad Complutense de Madrid, España.

${ }^{3}$ Instituto de Ciencias Forenses de la Universidad Autónoma de Madrid, España.

${ }^{4}$ Universidad Nacional de Educación a Distancia (UNED), España.

Recibido: 06 octubre de 2017.

Aceptado: 22 de marzo de 2018. 
bloqueando así las posibilidades de ayuda. Se encontró dependencia entre la estimación de credibilidad de su testimonio y la posibilidad de ser víctima de trata. El $94.4 \%$ de las mujeres cuyo testimonio resultó creíble no se consideró víctima de trata; el $72.8 \%$ de las estimadas como no creíbles fueron consideradas víctimas de trata. Según los entrevistadores, la posibilidad de que las entrevistadas estén siendo víctimas de trata dependió, en parte, de su aislamiento social, miedo o preocupación mostrado durante la entrevista, poca colaboración, evitar temas relevantes o mostrarse susceptible. Todo ello permite alertar sobre la situación de vulnerabilidad de las mujeres entrevistadas.

Palabras clave: Prostitución; Trata de personas; Explotación sexual; Mujer; Víctima.

\begin{abstract}
The objective of the present study was to carry out a study on the situation of women practicing prostitution in the community of Madrid (Spain), within the specific context of strip clubs and the possibility of them being victims of human trafficking networks. A total of 180 interviews were conducted on women practicing prostitution in strip clubs across the periphery of Madrid. The results analyzed show that, on average, women who engage in prostitution are 32.57 years of age; from Latin America, Europe or, from Africa (less frequently); and have generally undertaken some type of study/course. Around $80.5 \%$ of these women reported having a family, indicating that approximately $68 \%$ had between 1 and 4 children. Moreover, the present study analyzed the questions answered or avoided by these women, concluding that most of them omit relevant information regarding questions about trafficking and exploitation indicators. This finding leads to the suspicion that these women could be instructed / taught not to facilitate sensitive information, thus blocking the possibilities of help. A relationship of dependence between the testimony's credibility estimation and the possibility of being a victim of human trafficking was found. A $94.4 \%$ of women whose testimony was believed to be credible were not considered to be victims of human trafficking; $72.8 \%$ of
\end{abstract}

those estimated as non-credible were considered to be victims of human trafficking. The possibility of the interviewees being victims of human trafficking, according to interviewers, depended on their social isolation, fear or concern shown throughout the interview, their lack of collaboration, and their avoidance of certain relevant issues or being susceptible. Overall, the present study alerts about the situation of vulnerability of the interviewed women.

Keywords: Prostitution; Human trafficking; Sexual exploitation; Woman; Victim.

\section{Introducción}

La prostitución es aquella actividad en la que se mantienen relaciones sexuales a cambio de dinero. No solo afecta a las mujeres y hay una gran variedad de tipos, incluida la trata de personas (Weitzer, 2005a). Esta actividad se suele abordar desde perspectivas ideológicas muy diferentes (Brents, 2016; Farley, 2005; Weitzer, 2005b) y ha dado lugar al menos a dos paradigmas: el paradigma de la opresión y el paradigma polimorfo. El último aborda la prostitución desde una perspectiva compleja y no alude solo a la prostitución forzada (Weitzer, 2010). Hay diversas circunstancias que llevan a una persona a ejercer la prostitución (Hounmenou, 2016; Lerum y Brents, 2016; Marcus, Horning, Curtis, Sanson y Thompson, 2014). Entre ellas, algunas incluyen carencias en la vida de estas personas, afectivas, sociales, económicas, profesionales o sanitarias. Intentando satisfacerlas, la persona queda atrapada en la prostitución si no dispone de otros medios. Por otro lado, existe una prostitución forzada por terceros enmarcada en la trata de personas, con privación de libertad para ejercerla o abandonarla de forma voluntaria (Verhoeven, 2016). En muchos casos, estas personas se encuentran inmersas en redes de prostitución desde menores (Morselli y Savoie-Gargiso, 2014), permaneciendo largo tiempo por coacciones de todo tipo.

El Protocolo de Palermo para prevenir, reprimir y sancionar la trata de personas, especialmente mujeres y niños, de la Convención Internacional contra el Crimen Organizado (Naciones Unidas, 2004), define la trata de personas como aquella que implica la captación, por redes organi- 
zadas o personas individuales (conocidas de la víctima o incluso familiares), el transporte-traslado dentro de las fronteras de un país o fuera de ellas (entre países de origen-tránsito-destino).

En la trata de personas con fines de explotación sexual, la captación de las víctimas se realiza a partir del engaño, la agresión, las amenazas físicas y psicológicas, y la adquisición de importantes deudas económicas (Baker, Dalla y Williamson, 2010; Raphael y Shapiro, 2004). La explotación sexual tiene un grave riesgo sobre la salud de las personas que la sufren, generando en muchos casos graves trastornos psicológicos (Abas et al., 2013; Cecchet y Thoburn, 2014). Un estudio, realizado en nueve países de cuatro continentes (Farley et al., 2004), mostró que las mujeres que ejercían la prostitución frecuentemente sufrían traumas múltiples, en general consistentes en agresiones físicas $(71 \%)$ y violaciones $(63 \%)$ y carecían de un hogar donde vivir $(75 \%)$, lo que les llevaba a querer dejar la prostitución $(75 \%)$, y a padecer trastornos psicológicos como estrés postraumático (68\%) o toxicomanías.

Según la Red Española Contra la Trata de Personas (2010), en España estas víctimas suelen ser mujeres de 18 a 40 años, en situación de vulnerabilidad socioeconómica, que tras ser engañadas vienen al país para ejercer la prostitución en clubes, pisos o en la calle, siendo obligadas a vivir en lugares establecidos por la red que les está explotando. El alto control que sufren y la privación de una regularidad administrativa, les impide poder desenvolverse en la sociedad de manera normalizada, fomentando su aislamiento e imposibilitando la búsqueda de ayuda para salir de esa situación de trata.

La prostitución en España es considerada una actividad legítima, aunque no está regulada por ninguna legislación, careciendo de protección social y laboral. No existen estadísticas fiables sobre las personas que se dedican a esta actividad, resultando difícil realizar estudios comparativos internacionales (Weitzer, 2015). Un estudio sobre las operaciones de la Guardia Civil durante 2014 en España (Guardia Civil, 2015), señala que durante ese año se realizaron 20 operaciones contra la trata de seres humanos con fines de explotación sexual, 42 de explotación sexual, 56 de explotación laboral (77.26 \% en lugares donde se ejerce la prostitución), y 13 de explotación sexual junto con ex- plotación laboral. En las 42 operaciones contra explotación sexual se detuvieron a 50 hombres (72\%) y 22 mujeres, liberándose a 209 víctimas, de las que 204 eran mujeres $(98 \%)$, de nacionalidad mayoritariamente rumana (42\%), dominicana (14\%) y colombiana $(10 \%)$.

El objetivo del presente trabajo fue la realización de un estudio exploratorio sobre la situación de las mujeres que ejercen la prostitución en la Comunidad de Madrid (España). El contexto analizado fueron locales de alterne y la posibilidad de encontrar víctimas de redes de trata de personas. Los objetivos específicos fueron: (i) Explorar los datos sociodemográficos de mujeres que ejercen prostitución en Madrid en locales de alterne; (ii) obtener una primera información sobre potenciales indicadores de explotación sexual y trata de personas; (iii) analizar las relaciones entre indicadores; (iv) analizar indicadores de credibilidad estimada del testimonio de las mujeres por parte de los entrevistadores.

\section{Método}

\section{Participantes}

Se realizaron 181 entrevistas a mujeres que ejercen la prostitución en trece clubs de alterne de seis localidades de la Comunidad de Madrid, desde noviembre de 2013 hasta noviembre de 2015, en colaboración con Policía Judicial de Madrid. Una de las participantes (española, de 63 años de edad) fue excluida de los análisis debido a que probablemente no ejercía la prostitución, sino que era una de las "gerentes" del club, aunque manifestó ser una trabajadora más. La participación de las entrevistadas fue voluntaria y a todas se les garantizó la protección y anonimato de sus datos personales, cumpliendo con la normativa de investigación bioética con seres humanos. La selección de la muestra fue incidental, no aleatoria y determinada por el tipo de locales en los que se desarrollaba la actividad. En todas las entrevistas participó personal de policía judicial, psicólogos y abogados con formación especializada en psicología forense.

\section{Instrumentos de evaluación}


Con el fin de averiguar la existencia de indicios de trata de personas con fines de explotación sexual se diseñó una entrevista semiestructurada ad hoc basada en los indicios enumerados en la Instrucción 1/2010 de la Secretaría de Estado de Seguridad (SES. Ministerio del Interior, 2010) y en la Directiva de Servicio de la DAO, 40/2009 de Actuación contra la trata de Seres Humanos. Esta regulación atenúa la dificultad de obtener información en este tipo de casos, donde debe mantenerse un equilibrio entre no influir en las respuestas con preguntas excesivamente cerradas y sugestivas y el uso de preguntas demasiado abiertas que den lugar a respuestas evasivas (Lindholm et al., 2015). La entrevista fomentó el relato libre, comenzando con preguntas abiertas para profundizar en los aspectos relatados por la mujer que ejerce la prostitución. Se agrupó en cuatro bloques: datos personales (edad, nacionalidad, nivel educativo y datos familiares), datos relacionados con circunstancias del viaje (motivo de la migración, medio de llegada, ruta para llegar a España o existencia de deudas), y condiciones de vida (club donde trabaja, tiempo que lleva allí y ejerciendo la prostitución, condiciones laborales, domicilio, libertad de movimientos, satisfacción con su situación actual y expectativas de futuro). Finalmente, los entrevistadores emitieron un juicio intuitivo sobre la credibilidad del testimonio de las mujeres entrevistadas, donde estimaron la sinceridad de las respuestas obtenidas y si las entrevistadas podrían ser víctimas de trata de acuerdo con los indicios enumerados en la Instrucción 1/2010 (Ministerio del Interior, 2010): documentación; conocimiento del idioma; tiene dinero; libertad de desplazamiento y de dejar la prostitución; aislamiento social; redes de apoyo; condiciones migratorias y deudas contraídas; ansiedad, miedo, temor; signos de maltrato físico; vive en el club o es transportada bajo vigilancia; miedo o preocupación por su familia; mala condición de salud en general, tanto física como psíquica. Los datos fueron recogidos literalmente durante las entrevistas y posteriormente fueron pasados a una base de datos, categorizados en función de las respuestas encontradas.

\section{Procedimiento}

Una de las principales estrategias legales seguidas en nuestro país para identificar a las víctimas potenciales de trata de seres humanos con fines de explotación sexual son las inspecciones a los clubs de alterne. En estas inspecciones participan las Fuerzas y Cuerpos de Seguridad del Estado, Inspección de Trabajo y diferentes organizaciones que luchan contra la trata de personas. Tienen como objetivo encontrar posibles irregularidades e ilegalidades. Durante una inspección, las autoridades registran y aseguran el local, identificando a todos los presentes. Después, Inspección de Trabajo comprueba las condiciones laborales de las mujeres que allí ejercen la prostitución, tras esto se realizan entrevistas individuales comprobando la no vulnerabilización de derechos de estas mujeres, así como la existencia o no de indicios de trata. Además, se les informa sobre las posibilidades de denunciar y de las organizaciones especializadas al respecto.

Los entrevistadores recibieron formación para realizar las entrevistas, para cumplir con la legislación española y europea y para enfrentarse a potenciales víctimas. Se les informó de las dinámicas y roles del personal que trabaja en clubs de alterne y sobre el trabajo en el club. Así, los entrevistadores podrían detectar respuestas o indicadores de que las mujeres entrevistadas pudieran ser víctimas de trata. La preparación duró 12 horas, repartidas en tres días. Los entrevistadores se identificaron como personal de apoyo a policía judicial, en su mayoría psicólogos y algún abogado. La mayoría de los entrevistadores fueron mujeres $(85 \%)$. En todo momento estuvieron acompañados por personal de policía judicial. 


\section{Resultados}

\section{Datos personales}

Los datos mostraron que la edad de las mujeres que ejercen la prostitución en locales de alterne está comprendida entre los 18 y 52 años. En esta muestra de 180 mujeres, a excepción de siete casos en los que no quisieron señalar la edad, la media fue 32.57 años $(D T=7.99)$. De las 173 mujeres que la facilitaron, el $47.4 \%$, tiene entre 18 y 30 años, el $31.1 \%$ entre los 31 y 40 años y mayor de 41 años el $19.6 \%$.

La Tabla 1 muestra la distribución por nacionalidades, en la muestra no participaron mujeres procedentes de Asia o Norteamérica.

Tabla 1

Origen de las mujeres entrevistas

\begin{tabular}{|c|c|c|c|}
\hline & & Frecuencia & Porcentaje \\
\hline \multirow{5}{*}{ Europa } & & 71 & 39.2 \\
\hline & Bulgaria & 2 & 1.1 \\
\hline & España & 4 & 2.2 \\
\hline & Polonia & 1 & 0.6 \\
\hline & Rumanía & 64 & 35.4 \\
\hline \multirow{5}{*}{ África } & & 11 & 6.1 \\
\hline & Argelia & 1 & 0.6 \\
\hline & Cabo Verde & 1 & 0.6 \\
\hline & Marruecos & 8 & 4.4 \\
\hline & Otros & 1 & 0.6 \\
\hline \multicolumn{2}{|c|}{ Latinoamérica } & 99 & 54.7 \\
\hline & Bolivia & 1 & 0.6 \\
\hline & Brasil & 12 & 6.6 \\
\hline & Colombia & 18 & 9.9 \\
\hline & Ecuador & 11 & 6.1 \\
\hline & El Salvador & 1 & 0.6 \\
\hline & Paraguay & 5 & 2.8 \\
\hline & Perú & 1 & 0.6 \\
\hline & R. Dominicana & 49 & 27.1 \\
\hline & Venezuela & 1 & 0.6 \\
\hline
\end{tabular}

Se encontró que la edad media de las mujeres procedentes de África era mayor (39.82 años, $D T=7.65$ ) que la de las mujeres latinoamericanas (34.67 años, $D T=7.87)$ y la de las europeas $(28.50, D T=6.13)$; $F(1,172)=20.298, p<.001, \eta^{2}=.193$.
Sobre su tiempo de permanencia en España, el 40.9\% no lo indicó, señalando que no lo recuerdan con exactitud, no lo desean indicar o han ido pasando por diferentes países desde que comenzaron a ejercer la prostitución. El tiempo medio de estancia en España de las mujeres emigrantes que aportó el dato fue de 8.10 años $(D T=4.76)$. En función de su origen, se encontró que las mujeres africanas eran las que llevaban un mayor tiempo de estancia en España (10.29 años, $D T=6.62)$, seguidas por las latinoamericanas $(9.33$ años, $D T=4.79)$ y las europeas $(6.05$ años, $D T=3.61) ; \quad F(1,103)=7.143$, $p<.001 . \eta^{2}=.124$. Menos del $4 \%$ reconoció haber llegado a España hace menos de un año, otro $8.3 \%$ dijo llevar de 1 a 3 años en nuestro país. Un $23.2 \%$ reconoció llevar en nuestro país entre 6 y 10 años y un $12.2 \%$ entre 11 y 15 años. Finalmente, un $7 \%$ señaló llevar más de 16 años.

La mayoría de las entrevistadas manifestaron tener estudios (ver Tabla 2).

Tabla 2

Nivel de estudios

\begin{tabular}{lcc}
\hline & Frecuencia & Porcentaje \\
\hline Ninguno & 10 & 5.5 \\
Primarios & 32 & 17.8 \\
Secundaria & 44 & 24.4 \\
Bachillerato & 51 & 28.3 \\
Superiores & 39 & 21.7 \\
No contesta & 5 & 2.8 \\
\hline
\end{tabular}

Un $80.5 \%$ de ellas dijo tener familia (ver Tabla 3 ). Se puede estimar que sólo el $41.1 \%$ de las mujeres tienen algún apoyo familiar, si consideramos familia a la pareja, los hermanos y los padres, estando la mayoría de ellas solas. Manifiestan estar aisladas socialmente 31 de las mujeres entrevistadas (17.2\%) y solo $75(41.7 \%)$ afirman mantener comunicación con su familia. 
Tabla 3

\section{Datos familiares}

\begin{tabular}{lcc}
\hline & Frecuencia & Porcentaje \\
\hline Tiene familia & 145 & 80.5 \\
No tiene familia & 25 & 13.9 \\
Estado civil & & \\
Con Pareja & 44 & 22.4 \\
Soltera & 70 & 38.9 \\
Divorciada/Separada/Viuda & 30 & 16.7 \\
No especifica & 36 & 20.0 \\
Hijos & & \\
Ninguno & 54 & 31.9 \\
Uno & 65 & 38.5 \\
Dos & 30 & 17.7 \\
Tres & 17 & 10.0 \\
Cuatro & 3 & 1.8 \\
Tienen hermanos & 23 & 12.8 \\
Mantienen contacto con sus & 9 & 39.1 \\
hermanos & & \\
Mantienen contacto con sus & 32 & 17.8 \\
padres & & \\
Apoyo familiar (pareja, & 74 & 41.1 \\
hermanos, padres) & & \\
\hline
\end{tabular}

\section{Circunstancias del viaje a España y motivos de la emigración}

Un 21.5\% no especificó cómo fueron las circunstancias del viaje a España. El 56.9 \% indicó haber llegado en avión y un $16.0 \%$ en autobús. El $5.6 \%$ restante dijo haber llegado en coche o usando inicialmente el avión junto a otros transportes como el tren o barco.

Un 43.1 \% indicaron haber llegado a España de forma directa, mientras que un $17.7 \%$ indicó haber hecho algún tipo de escala o haber pasado por varios países antes de llegar.

Sobre los motivos para migrar, la mayoría informó que vino para buscar trabajo y mejorar su situación $(70.7 \%)$, un $16.6 \%$ por motivos familiares, por turismo $(4.4 \%)$ o para formarse $(1.1 \%)$. Sólo un $2.2 \%$ reconoció haber migrado para ejercer la prostitución. Un 5\% prefirió no señalar los motivos.
La Tabla 4 recoge las respuestas sobre el pago del viaje para llegar a España.

Tabla 4

Fuente de financiación para viajar a España

\begin{tabular}{lcc}
\hline & Frecuencia & Porcentaje \\
\hline No se indica & 125 & 69.4 \\
Ella misma & 36 & 20.0 \\
Familiar & 12 & 6.7 \\
Amigo/a & 4 & 2.2 \\
Desconocido & 2 & 1.1 \\
La empresa & 1 & 0.5 \\
Total & $\mathbf{1 8 0}$ & $\mathbf{1 0 0}$ \\
\hline
\end{tabular}

La Tabla 5 resume los datos relacionados con las ofertas de trabajo, antes de viajar y la primera ocupación al llegar. Se observaron diferencias en las ofertas laborales que motivaron su migración en función de su origen, $\chi^{2}(26, N=180)=55.404, p<.001$. Las mujeres latinoamericanas, en general, llegan a España sin ofertas de trabajo $(72.7 \%)$ y por motivos familiares o personales, solo el $7.1 \%$ vino sabiendo que iban a dedicarse a la prostitución. Las mujeres africanas, también llegan sin ofertas de trabajo en su mayoría (54.5\%), y solo una llegó con una oferta para ejercer la prostitución. Las mujeres europeas, en la mayoría de las ocasiones tampoco tuvieron ofertas de trabajo (55.7\%), pero en éstas son las ofertas para ejercer la prostitución las que prevalecen por encima del resto, habiendo emigrado para ejercerla el $24.3 \%$. Así pues, la oferta previa para ejercer la prostitución dependía de la procedencia de las mujeres entrevistadas, $\chi^{2}(4$, $N=180)=12.409, p<.05$.

Respecto a su primera ocupación laboral en España según origen (ver Tabla 5), las europeas se habrían dedicado mayoritariamente a ejercer la prostitución (48.6\%), las latinoamericanas a otras actividades $(26.3 \%)$ y a la prostitución $(23.2 \%)$, y las africanas a otras actividades (36.4\%) o a nada $(27.3 \%)$. Así, su primera actividad dependía de su procedencia, $\chi^{2}(8, N=180)=18.994$, $p<0.05$. 
Tabla 5

Oferta previa de trabajo y primera ocupación en España en función de su procedencia

\begin{tabular}{|c|c|c|c|c|c|c|}
\hline & \multicolumn{2}{|c|}{ Europa $(\mathrm{N}=70)$} & \multicolumn{2}{|c|}{ Latinoamérica $(\mathrm{N}=99)$} & \multicolumn{2}{|c|}{ África $(\mathrm{N}=11)$} \\
\hline & Frecuencia & $\%$ & Frecuencia & $\%$ & Frecuencia & $\%$ \\
\hline \multicolumn{7}{|c|}{ Oferta previa } \\
\hline Prostitución & 17 & 24.3 & 7 & 7.1 & 2 & 18.2 \\
\hline Camarera & 1 & 1.4 & 2 & 2.0 & 0 & 0 \\
\hline Ninguna & 39 & 55.7 & 72 & 72.7 & 6 & 54.5 \\
\hline Otras & 4 & 5.7 & 9 & 9.1 & 2 & 18.2 \\
\hline No contesta & 9 & 12.9 & 9 & 9.1 & 1 & 9.1 \\
\hline \multicolumn{7}{|c|}{ Primera dedicación } \\
\hline Prostitución & 34 & 48.6 & 23 & 23.2 & 2 & 18.2 \\
\hline Camarera & 13 & 18.6 & 9 & 9.1 & 2 & 18.2 \\
\hline Ninguno & 5 & 7.1 & 17 & 17.2 & 3 & 27.3 \\
\hline Otras & 10 & 14.3 & 26 & 26.3 & 4 & 36.4 \\
\hline No contesta & 13 & 18.6 & 24 & 24.2 & 2 & 18.2 \\
\hline
\end{tabular}

La diferencia entre las mujeres que venían con una oferta para ejercer la prostitución y las que realizaron este trabajo al llegar, indica que al menos un $22 \%$ fueron engañadas con la oferta laboral. Un $36.9 \%$ si se considera también a las que afirman haberse dedicado a ser camareras o trabajadoras de un club, cifras que podrían ser mayores si tenemos en cuenta que muchas de las $39 \mathrm{mu}-$ jeres que no informan de a qué se dedicaron al llegar a España, también podrían estar ejerciendo la prostitución.

Por otra parte, un $29.3 \%$ dijo haber conseguido este trabajo gracias a algún conocido, familiar o amigo. Las mujeres que habían trabajado en varios clubs declararon tener la intención de dejar la prostitución y encontrar otro trabajo.

\section{Condiciones de vida y laborales}

Quince de las mujeres entrevistadas (8.3\%) afirman vivir solas, 139 (77.2\%) no informan con quién viven, 72 (40\%) dicen dormir en un lugar distinto al club de alterne y $60(33.3 \%)$ reconocen dormir allí.

De las 180 mujeres, 30 (16.67\%) dicen que alguien relacionado con el trabajo las recoge y las lleva al club, y $133(73.89 \%)$ no informan cómo llegan al trabajo. No obstante, solo 28 de las 72 mujeres que dicen dormir en un lugar distinto al club informan cómo llegan al trabajo y de éstas, 8 (4.44\% del total) dicen ir sin compañía de alguien relacionado con el club y $19(11.11 \%)$ afirman ir solas. Ninguna de las 48 mujeres que evitaron contestar a la pregunta sobre dónde vivían, informa cómo se traslada hasta el trabajo.

Dormir en el club dependía de si se dedicaban exclusivamente a la prostitución, $\chi^{2}(4, N=180)=14.705$, $p<.005$. Duerme en el club el $51.7 \%$ de las que se dedican en exclusiva a la prostitución, frente a un $36.7 \%$ de las mujeres que la ejercían a tiempo parcial.

Cuando se pregunta si pueden cambiar de domicilio libremente solo siete $(3.9 \%)$ contestan afirmativamente. Aunque 164 mujeres $(91.1 \%)$ manifiestan tener libertad de movimientos, $30(16.67 \%)$ dicen que las acompañan cuando quieren salir, $148(82.22 \%)$ no contesta y solo dos $(1.1 \%)$ afirman que pueden salir cuando quieren.

Los datos muestran que vivir sola o en compañía es independiente de la edad $[F(2,98)=0.167, p=$ n.s., $\left.\eta^{2}=.003\right]$, el tiempo de estancia en España $\left[F(2,98)=1.288, p=\right.$ n.s., $\left.\eta^{2}=.026\right]$, o el tiempo dedicado a ejercer la prostitución $[F(2,79)=1.060, p=$ n.s., $\left.\eta^{2}=.027\right]$. 
Aunque 79 (43.9 \%) no aportan información sobre sus condiciones laborales, $11(6.1 \%)$ dicen que es su primer día o acaban de empezar y 39 (21.7\%) que ejercen solo desde hace unas semanas. Por otro lado, 62 mujeres (34.4\%) aportan más información sobre el tiempo dedicado a la prostitución y su permanencia en el club actual. En estas últimas, el tiempo medio de dedicación a la prostitución es de 2.14 años $(D T=2.77)$, con un mínimo de un mes y un máximo de 13 años. El tiempo de permanencia en el actual club es de 1.53 años $(D T=2.19)$, un mínimo de un día y un máximo de 11 años. La procedencia de las mujeres entrevistadas no muestra diferencias con respecto al tiempo de ejercicio de la prostitución, $F(2,61)=0.558$, $p=$ n.s., $\eta^{2}=.022$; ni el tiempo de estancia en el club actual, $F(2,61)=0.813, p=$ n.s., $\eta^{2}=.032$.

Llegar a España con una oferta para dedicarse a la prostitución, tampoco implica más tiempo de dedicación y estancia en el club actual, en comparación con las mujeres que no sabían a qué se iban a dedicar, $F(2,61)=2.233$, $p=$ n.s., $\eta^{2}=.076$ y $F(2,61)=2.569, p=$ n.s., $\eta^{2}=.087$, respectivamente. 98 mujeres $(54.4 \%)$ afirmaron que se dedicaban a la prostitución y a otros trabajos. Respecto a si habían trabajado antes en otros clubs, $117(65.0 \%)$ no contestaron, $15(8.3 \%)$ contestaron negativamente y 48 $(26.67 \%)$ lo afirmaron.

La dedicación en exclusiva a la prostitución dependía de su procedencia, $\chi^{2}(4, N=180)=18.413, p<.001$. Se dedicaban en exclusiva a esta actividad el $51.4 \%$ de las europeas, el $22.2 \%$ de las latinoamericanas y el $27.3 \%$ de las africanas.

Solo nueve (5.0 \%) afirmaron estar satisfechas con las condiciones de trabajo. No obstante, el $68.3 \%$ manifestó que pensaba dejar la prostitución en un futuro y 42 $(23.3 \%)$ manifestaron su intención de seguir ejerciéndola. Un $8.9 \%$ dijo estar buscando otro empleo en ese momento o estar a la espera de posibles entrevistas de trabajo, aunque un $84.4 \%$ manifestaron no estar buscando otra cosa.

Querer dejar la prostitución dependía de su procedencia, $\chi^{2}(4, N=180)=19.181, p<.001$. No manifestó querer dejarlo el $40 \%$ de las europeas, el $18.2 \%$ de las africanas y el $8.1 \%$ de las latinoamericanas.
Dedicarse en exclusiva a la prostitución depende de haber llegado con una oferta relacionada con su ejercicio, $\chi^{2}(4, N=180)=51.020, p<.001$. El $92 \%$ que llegó para ejercer se dedica de forma exclusiva, por solo el $24 \%$ de las que llegó por otros motivos. Querer dejar ese trabajo igualmente depende de venir con una oferta previa, $\chi^{2}(4$, $N=180)=16.697, p<.005$. El $72.6 \%$ que llegó para dedicarse a otras actividades quiere dejarlo, frente el $56 \%$ que vino para eso.

De las mujeres extranjeras, a un $21 \%$ le gustaría volver a su país. A pesar de que un $32.6 \%$ no especificó su jornada laboral, el $35.9 \%$ dijo poder ir a trabajar cuando quisiera y un $22.8 \%$ informó tener jornadas laborales de más de seis horas seguidas. Así mismo, el $93.9 \%$ dijo no sentirse obligada a permanecer en el trabajo. El $70.2 \%$ consideró que podía faltar a causa de enfermedades u otras razones.

\section{Percepción intuitiva de la credibilidad de los testimonios}

Debido a las características de este tipo de entrevistas, que reflejan las respuestas explícitas, pero no otros indicadores que rodean a la entrevista (comportamiento no verbal individual y grupal, conductas que señalen temor o ser observadas), se solicitó a los entrevistadores que emitieran un juicio intuitivo de credibilidad sobre la información aportada por las 180 mujeres una vez terminada la entrevista. Debían responder a una pregunta basada en el análisis general de la entrevista y contexto que rodeó a la misma: “el relato de la mujer fue creíble / no creíble?”. Tras esta pregunta debían responder su percepción sobre si la mujer entrevistada pudiera ser víctima de trata, con las posibilidades de respuesta "si/no", " ¿La mujer entrevistada es víctima de trata de personas?" Las Tablas 6 y 7 resumen las conclusiones de los entrevistadores sobre la posibilidad de que estas mujeres hayan aportado un testimonio creíble, sin contradicciones y la valoración inicial sobre posibles indicios o situaciones que convierten a la mujer entrevistada en una víctima potencial de trata de personas con fines de explotación sexual. 
Tabla 6

Credibilidad percibida de su testimonio sobre ausencia de coacciones para ejercer la prostitución

\begin{tabular}{lcc}
\hline & \multicolumn{2}{c}{ Testimonio potencialmente creible } \\
\hline & Frecuencia & Porcentaje \\
SI & 116 & 64.4 \\
NO & 64 & 35.6 \\
Total & 180 & 100 \\
\hline
\end{tabular}

Tabla 7

Presencia de indicadores de que la mujer entrevistada podría estar siendo vícitma de trata de personas

\begin{tabular}{lrr}
\hline & Posibilidad de ser víctima de trata \\
\hline & Frecuencia & Porcentaje \\
SI & 81 & 45.5 \\
NO & 99 & 55.5 \\
Total & $\mathbf{1 8 0}$ & $\mathbf{1 0 0}$ \\
\hline
\end{tabular}

Se encontró una relación estadística de dependencia entre la estimación intuitiva de credibilidad de su testimonio y la posibilidad de ser víctima de trata, $\chi^{2}(1$, $N=180)=89.346, p<.001$. El $94.4 \%$ de las mujeres cuyo testimonio se percibió como creíble, no se consideró víctima de trata; el $72.8 \%$ de las estimadas como no creíbles, fueron consideradas víctimas de trata.

El análisis mostró que la posibilidad de que las entrevistadas estén siendo víctimas de trata, según los entrevistadores, dependía de su aislamiento social, $\chi^{2}(2$, $N=180)=5.898, p=.05$, miedo o preocupación mostrado durante la entrevista, $\chi^{2}(2, N=180)=7.501, p<.05$, poca colaboración, $\chi^{2}(2, N=180)=10.233, p<.01$, evitar tratar temas relevantes, $\chi^{2}(2, N=180)=14.096, p=.001$, mostrarse susceptible, $\chi^{2}(2, N=180)=1.021, p<.001$, informa que no es golpeada, $\chi^{2}(2, N=180)=4.284$, $p<.05$, no sufre abusos físicos, $\chi^{2}(2, N=180)=4.284$, $p<.05$, o sexuales, $\chi^{2}(2, N=180)=4.284, p<.05$, hace uso o dispone de su propio dinero, $\chi^{2}(1$, $N=180)=19.733, p<.001$, está buscando otro trabajo, $\chi^{2}(2, N=180)=8.035, p<.05$, y tiene libertad para dejar la prostitución, $\chi^{2}(2, N=180)=11.579, p<.01$.
El $64 \%$ de las mujeres que estaban aisladas socialmente fueron percibidas como víctimas de trata, por solo $42.5 \%$ de las que no lo estaban. De las siete mujeres que se mostraron preocupadas o temerosas seis fueron percibidas como víctimas de trata. Al $100 \%$ de las que colaboraron, no se las consideró víctimas, mientras que al $7.4 \%$ que no colabora, se sospechó que si lo eran. El 91.7 \% que evitó tratar algunos temas fue considerada víctima de trata, frente al $41.0 \%$ que no lo evitó. El $80 \%$ que se mostró susceptible fue considerada víctima, por un $39.9 \%$ que no se mostraron así. El 57.2 \% que informó que no es golpeada o no sufre abusos físicos o sexuales no es considerada víctima. El $86.8 \%$ que manifiesta hacer uso de su propio dinero, y el $87.5 \%$ que afirma estar buscando otro trabajo no son consideradas víctimas. Todas las que afirman que no tienen libertad para dejar la prostitución, fueron consideradas víctimas.

La credibilidad estimada dependió de los siguientes indicadores: presentar documentación, $\chi^{2}(4$, $N=180)=10.793, p<.05$, mostrarse susceptible durante la entrevista, $\chi^{2}(2, N=180)=23.728, p<.001$, no colaborar, $\chi^{2}(2, N=180)=10.041, p<.01$, evitar tratar temas relevantes, $\chi^{2}(2, N=180)=21.806, p<.001$, afirmar explícitamente no recibir golpes, abusos físicos o sexuales, $\chi^{2}(1, N=180)=5.469, p<.05$, decir querer dejar la prostitución, $\chi^{2}(2, N=180)=9.435, p<.01$, tener deudas de viaje, $\chi^{2}(3, N=180)=9.161, p<.05$, señalar tener libertad para dejar la prostitución, $\chi^{2}(2, N=180)=12.990$, $p<.01$, para faltar algún día al trabajo, $\chi^{2}(3$, $N=180)=9.665, p<.05$, tener libertad de movimientos, $\chi^{2}(5, N=180)=13.627, p<.05$, manejar su propio dinero, $\chi^{2}(1, N=180)=16.084, p<.001$, y buscar trabajo, $\chi^{2}(2, N=180)=8.824, p<.05$.

El $66.7 \%$ de las mujeres que no presentó documentación, fue considerada como no creíble frente al $30.5 \%$ que sí la presentó. El 80 \% que muestra susceptibilidad es considerada no creíble. El $83.3 \%$ de las que no colabora y el $91.7 \%$ de las que evita tratar temas sensibles es considerada no creíble. El $66.9 \%$ de las que colabora y el $69.3 \%$ de las que no rehúsa tratar algunos temas es considerada creíble. El 66.9 \% de las que manifiesta explícitamente no sufrir golpes, abusos físicos o sexuales es considerada creíble. El $70.7 \%$ que afirma querer dejar la prostitución 
es considerada creíble, frente al $57.1 \%$ que indica no querer dejarlo. El $75 \%$ que afirma tener deudas por el viaje a España es considerada creíble, frente al $67.9 \%$ de las que dice no tenerlas. De las mujeres que dicen tener libertad para dejar la prostitución, un $67.9 \%$ es creíble, por un $32.1 \%$ que no lo es. Cuando afirman poder faltar al trabajo, el $71 \%$ es creíble por un $29 \%$ que no lo es. De las que dicen tener libertad de movimientos, el $67.7 \%$ es creíble por el $32.3 \%$ que no. Respecto al manejo de su propio dinero, el $7.9 \%$ no es creíble por el $92.1 \%$ que sí. El $93.5 \%$ que señala buscar trabajo y el $67.3 \%$ que dice tener libertad para dejar la prostitución es creíble, frente al $63.2 \%$ que dice que no lo está buscando y el $33.3 \%$ que señala no tener libertad.

\section{Discusión y conclusiones}

En este trabajo se evaluaron de manera exploratoria y descriptiva los indicadores de trata de personas en mujeres que ejercen la prostitución en locales de alterne, según la Instrucción 1/2010 de la SES y en la Directiva de Servicio de la DAO, 40/2009 de Actuación contra la Trata de Seres Humanos.

Los datos permiten concluir que las mujeres tenían aproximadamente treinta años y eran de diversas nacionalidades, siendo en su mayoría de Europa y Latinoamérica. Llama la atención que no se encontraron mujeres asiáticas ni apenas de origen africano (solo $5.5 \%$ del total), por lo que es posible que las mujeres de dichos continentes ejerzan la prostitución mayoritariamente en contextos distintos a los clubes de alterne. Por otros estudios, parece que las mujeres africanas ejercen la prostitución de calle, mientras que las mujeres asiáticas lo harían en pisos clandestinos (Guardia Civil, 2015).

Sobre el objetivo específico de indicadores de explotación sexual y trata, parece desprenderse de los datos que las mujeres entrevistadas omiten información relevante sobre muchas de las preguntas realizadas sobre los indicadores de trata y explotación, lo que sugiere que pudieran estar instruidas para no facilitar información sensible, blo-

\footnotetext{
1 Agradecemos a la profesora de la UNED Laura Quintanilla su

sugerencia para esta interpretación y la referencia a este trabajo.
}

queando así las posibilidades de ayuda. Por ejemplo, el $77 \%$ de las mujeres no informó de la situación en la que viven, el $88 \%$ de las mujeres no contestó a la pregunta sobre su libertad de movimientos, se niegan coacciones, o se omite información sobre sus trabajos anteriores y detalles de la situación actual. Todos estos datos que se ocultan, en su conjunto, pueden considerarse indicios sobre la dificultad de la situación actual de las mujeres entrevistadas.

Respecto a esto último, se encontró una relación estadística de dependencia entre la estimación de credibilidad percibida por los entrevistadores de su testimonio y la posibilidad de ser víctima de trata. Esta valoración por parte de los entrevistadores dependía, entre otros indicios, de la falta de colaboración con la entrevista, considerando por tanto sus testimonios potencialmente no creíbles. El mayor porcentaje de respuestas omitidas se encontró precisamente en las variables "sensibles", igual que otros trabajos han encontrado en estudios sobre la misma temática (Lindholm et al., 2015). Siguiendo a Callejo (2002) ${ }^{1}$, comprobamos que estos silencios de las entrevistadas nos han dado unos no-datos como un espejo "en negativo". Callejo defiende la entrevista en profundidad como una especie de "confesión" del entrevistado, siendo más probable que se confiese aquello que forma parte de normas aceptables por la sociedad. Por ello, los silencios no se producen porque la información se ignore, sino que es un "callar activo" de lo que no se quiere expresar. Así pues, el presente trabajo facilita también datos sobre silencios, que constituye un material empírico igualmente valioso. Por otro lado, el presente trabajo aporta datos sobre indicios de credibilidad que han resultado estadísticamente significativos y que pueden resultar de ayuda para futuras investigaciones.

Los estudios sobre esta problemática son imprescindibles para dar un primer paso y acercarse a aquellas mujeres víctimas potenciales de trata y explotación sexual. Por todo ello, es importante la respuesta de las Fuerzas y Cuerpos de Seguridad para la prevención, represión e investigación de estos delitos, tanto desde la labor preventiva y de detección de víctimas en las distintas inspecciones como a través de las distintas operaciones de investiga- 
ción. En este trabajo nos hemos centrado en un tipo muy concreto de prostitución, la que se ejerce en locales de alterne en la periferia de Madrid. Los resultados no son extrapolables directamente a otros tipos de prostitución, donde se podrían encontrar otros rangos de edad y nacionalidades de procedencia, e idiosincrasias particulares por razón de raza o religión. Un ejemplo, lo tendríamos con las mujeres nigerianas que son captadas a través de amenazas de vudú y que durante años mantienen el control sobre ellas por el miedo a que ejerzan daño a sus familiares a través de esta práctica de magia (Baarda, 2016).

Este trabajo presenta algunas limitaciones, entre las que cabe mencionar la representatividad de la muestra. Se trata de un estudio incidental con una selección muestral no aleatoria y restringida a un contexto y área geográfica particular. Es importante señalar que hubo un elevado porcentaje de mujeres entrevistadas que, a juicio de los entrevistadores, no facilitaron un testimonio creíble, al negar sistemáticamente o no contestar las preguntas que permitirían avalar un posible abuso o una situación de trata de personas. Esto lleva a considerar los presentes resultados con cautela. Futuros trabajos con muestras más amplias y con una estimación aleatoria y representativa podrían arrojar más contundencia a los datos hallados. No obstante, y a pesar de las limitaciones señaladas, estos resultados permiten una primera aproximación a una realidad de difícil acceso y escasamente investigada.

En entrevistas con ausencia de indicios claros de delito no se pueden activar medidas de mayor calado para la lucha contra la explotación sexual o trata de seres humanos. Sin embargo, las entrevistas en el contexto de las inspecciones impulsan un contacto y conversaciones con estas mujeres que, al menos, permiten informarles sobre posibles alternativas y facilitar el conocimiento de las instituciones y asociaciones que podrían ayudar a estas mujeres a cambiar su situación o, al menos, conocer las posibilidades de ayuda con las que cuentan y alternativas al ejercicio de la prostitución.

\section{Referencias}

Abas, M., Ostrovschi, N. V., Prince, M., Gorceag, V. I., Trigub, C. y Oram, S. (2013). Risk Factors for Mental Disorders in Women Survivors of Human Trafficking: A Historical Cohort Study. $B M C$ Psychiatry, 13(204), 1-11. https://doi.org/10.1186/1471-244X-13-204

Baarda, C. S. (2016). Human Trafficking for Sexual Exploitation from Nigeria into Western Europe: The role of voodoo Rituals in the Functioning of a Criminal Network. European Journal of Criminology, 13(2), 257-273. https://doi.org/10.1177/1477370815617188

Baker, L. M., Dalla, R. L. y Williamson, C. (2010). Exiting Prostitution: An Integrated Model. Violence Against Women, 16(5), 579-600. https://doi.org/10.1177/1077801210367643

Brents, B. G. (2016). Neoliberalism's market morality and heteroflexibility: Protectionist and free market discourses in debates for legal prostitution. Sexuality Research and Social Policy, 13(4), 402416. https://doi.org/10.1007/s13178-016-0250-0

Callejo, J. (2002). Observación, Entrevista y Grupo de Discusión: el Silencio de tres prácticas de investigación [Observation, Interview and Discussion Group: The Silence of Three Research Practices]. Revista Española de Salud Pública, 76, 409-422. Recuperado de http://scielo.isciii.es/scielo.php?script=sci_arttext \&pid $=$ S1 135-57272002000500004

Cecchet, S. J. y Thoburn, J. (2014). The psychological experience of child and adolescent sex trafficking in the United States: Trauma and resilience in survivors. Psychological Trauma: Theory, Research, Practice, and Policy, 6(5), 482-493. https://doi.org/10.1037/a0035763

Farley, M. (2005). Prostitution Harms Women even if Indoors: Reply to Weitzer. Violence Against 
Women, 11(7), 950-964. https://doi.org/10.1177/1077801205276987

Farley, M., Cotton, A., Lynne, J., Zumbeck, S., Spiwak, F., Reyes, M. E y Sezgin, U. (2004). Prostitution and Trafficking in Nine Countries: An Update on Violence and Posttraumatic Stress Disorder. Journal of Trauma Practice, 2(3-4), 33-74. https://doi.org/10.1300/J189v02n03_03.

Guardia Civil (2015). Informe Criminológico 2/2015. La Trata de Seres Humanos y conductas afines en España [Human Trafficking and Related Behaviours in Spain]. Madrid, España: Ministerio del Interior.

Hounmenou, C. (2016). Exploring Child Prostitution in a major city in the West African region. Child Abuse \& Neglect, 59, 26-35. https://doi.org/10.1016/j.chiabu.2016.07.003

Lerum, K. y Brents, B. G. (2016). Sociological Perspectives on Sex Work and Human Trafficking. Sociological Perspectives, 59(1), 17-26. https://doi.org/10.1177/0731121416628550

Lindholm, J., Cederborg, A. C. y Alm, C. (2015). Adolescent Girls Exploited in the Sex Trade: Informativeness and Evasiveness in Investigative interviews. Police Practice and Research, 16(3), 197-210.

https://doi.org/10.1080/15614263.2014.880839

Marcus, A., Horning, A., Curtis, R., Sanson, J. y Thompson, E. (2014). Conflict and Agency among Sex Workers and Pimps: A Closer Look at Domestic Minor Sex Trafficking. The Annals of the American Academy of Political and Social Science, 653(1) 225-246. https://doi.org/10.1177/0002716214521993

Ministerio del Interior (2010). Instrucción 1/2010 de la Secretaría de Estado de Seguridad sobre aplicación transitoria, a las víctimas de la trata de seres humanos, del artículo 59.bis de la Ley Orgánica 4/2000, de 11 de enero [Instruction
1/2010 of the Secretary of State for Security on the Transitional Application to Victims of Human Trafficking, of Article 59.bis of Organic Law 4/2000, of 11th January] Madrid, España: Ministerio del Interior.

Morselli, C. y Savoie-Gargiso, I. (2014). Coercion, control, and cooperation in a prostitution ring. The Annals of the American Academy of Political and Social Science, 653(1), 247-265. https://doi.org/10.1177/0002716214521995

Naciones Unidas (2004). Convención de las Naciones Unidas contra la delincuencia organizada transnacional y sus protocolos [United Nations Convention Against Transnational Organized Crime And Its Protocols]. Recuperado de http://www.unodc.org/documents/treaties/UNTOC /Publications/TOC\%20Convention/TOCebooks.pdf

Raphael, J. y Shapiro, D. L. (2004). Violence in Indoor and Outdoor Prostitution Venues. Violence Against Women, 10(2), 126-139. https://doi.org/10.1177/1077801203260529

Red Española Contra la Trata de Personas (2010). La trata de personas en el mundo y en España [Human Trafficking Worldwide and in Spain]. Recuperado de

http://areadesociales.weebly.com/uploads/1/0/8/8/ 10886535/panorama.pdf.

Verhoeven, M. (2016). Sex Work Realities versus Government Policies: Meanings of AntiTrafficking Initiatives for Sex Workers in the Netherlands. Sexuality Research and Social Policy, 1-10 https://doi.org/10.1007/s13178-016-0264-7

Weitzer, R. (2005a). New Directions in Research on Prostitution. Crime, Law and Social Change, 43(45), 211-235. https://doi.org/10.1007/s10611-005$1735-6$

Weitzer, R. (2005b). Flawed Theory and Method in Studies of Prostitution. Violence Against Women, 
11(7),

934-949.

https://doi.org/10.1177/1077801205276986.

Weitzer, R. (2010). The mythology of prostitution:

Advocacy research and public policy. Sexuality

Research and Social Policy, 7(1), 15-29.

https://doi.org/10.1007/s13178-010-0002-5
Weitzer, R. (2015). Researching prostitution and sex trafficking comparatively. Sexuality Research and Social Policy, 12(2), 81-91. https://doi.org/10.1007/s13178-014-0168-3

\title{
INDICATORS OF TRAFFICKING IN WOMEN PRACTICING PROSTITUTION IN STRIP CLUBS IN THE COMMUNITY OF MADRID (SPAIN)
}

\author{
Eva A. SILVA ${ }^{1}$, ANTONIO L. MANZANERO ${ }^{2}$, \\ GARAZI BENGOA ${ }^{3}$ Y MARÍA JOSÉ CONTRERAS ${ }^{4}$
}

\section{EXTENDED SUMMARY}

There are various circumstances that can lead a woman to engage in prostitution, many of them related to different needs that may be lacking in this person's life, such as affective, social, economic, professional or health deficiencies. In general, it is a disadvantage for this woman, who becomes trapped in that activity if she lacks other means by which to obtain the necessary economic benefit.

Regardless of the social and cultural problem that seeing this practice as something natural entails, the relevant issue of the present study is sexual exploitation and the serious risk that this activity has on the health of women practicing prostitution. A study carried out across nine countries in four continents (Farley et al., 2004) showed that women who frequently practiced prostitution suffered multiple trauma, generally consisting of physical aggression $(71 \%)$ and rape $(63 \%)$ and had nowhere to live (75\%), which leads them to want to stop practicing pros- titution (75\%), and to suffer psychological disorders such as post-traumatic stress $(68 \%)$ and drug addiction.

Furthermore, a recent study (Guardia Civil, 2015) on Civil Guard operations in Spain in 2014 states that, during that year, 20 operations were carried out against human trafficking with the purpose of sexual exploitation, 42 in cases of sexual exploitation, 56 in cases of work exploitation $(77.26 \%$ in places where prostitution is practiced), and 13 operations in cases of sexual exploitation with work exploitation. In cases of human trafficking for the purpose of sexual exploitation, the victims were mainly from Romania (46\%), Bulgaria (13\%) and Spain (9\%), of which $95 \%$ were women, most of them aged between 20 and 30 years. The traffickers were Romanian $(47 \%)$, Spanish $(20 \%)$ and Bulgarian (14\%).

The general objective of the present work was to carry out a descriptive study of the situation of women practicing prostitution in the community of Madrid (Spain), in the specific context of strip clubs, and the possibility of them being victims of human trafficking networks. After carry- 
ing out a literature review, the social exclusion and legal vacuum in which these women who practice prostitution may be immersed in becomes apparent. Allegedly, the women being interviewed engage in prostitution on a voluntary basis, although most suggest that prostitution is not their life project, but rather that they are drawn into this reality due to various situations of vulnerability (debts, threats, gender violence, etc.).

Thus, the present study analyzed a total of 180 interviews with women practicing prostitution in strip clubs across six localities of the Community of Madrid between November 2013 and November 2015 that were provided by the Judicial Police of Madrid. All the interviewees were guaranteed the protection and anonymity of their personal data, complying with the bioethics regulations on research with human beings.

The interview sought to encourage free narrative, starting with open-ended questions, in order to aid further deepening into certain aspects reported by women practicing prostitution. Questions were grouped into four blocks: personal data, data related to travel circumstances, living conditions and credibility of the testimony, which was intuitively judged by the interviewers.

The data showed that the age of women who practice prostitution is between 18 and 52 years. In this sample of 180 women, with the exception of seven cases in which the women did not want to specify their age, the mean age was 32.57 years $(S D=7.99)$. Of the 173 women who did facilitate their age, $47.4 \%$ were between 18 and 30 years old, $31.1 \%$ were between 31 and 40 years old and $19.6 \%$ were over 41 years of age.

In relation to the country of origin of the interviewed women, none originated from Asia or North America. Of the 180 women interviewed, 4 (2.2\%) were of Spanish origin and $12(6.6 \%)$ had dual nationality. The majority of women were Latin American (54.7\%), Dominican (27.1\%), Colombian (9.9\%), Brazilian (6.6\%) and Ecuadorian $(6.1 \%)$. A $37.1 \%$ of women came from Eastern European countries, with the Romanian nationality being the most frequent (35.4\%). Only 11 women were of African origin $(6.1 \%)$.
Based on their origin, it was found that the mean age of women from Africa was higher (39.82 years, $S D=7.65$ ) than that of women from Latin America (34.67 years, $S D=7.87)$ and that of women from Europe (28.50, $D T=6.13) ; F(1,172)=20.298, p<.001, \eta^{2}=.193$.

Regarding their arrival to Spain, $40.9 \%$ did not indicate when it occurred, noting that they did not remember exactly, did not wish to indicate it or that they had moved around different countries since they began to exercise prostitution. The mean time of stay in Spain of the immigrant women that reported this information was of 8.10 years $(S D=4.76)$.

Furthermore, the majority of the women interviewed indicated having undertaken some type of studies, with only 5 women refraining from answering this question.

In relation to family, an $80.5 \%$ of these women reported having a family. Regarding the number of children, $169(93.9 \%)$ women answered this question. Of these, 54 women $(31.9 \%)$ stated that they did not have any children and $115(68.0 \%)$ stated having between 1 and 4 children.

Likewise, it can be estimated that only $41.1 \%$ of the women had some family support, if the woman's partner, siblings and parents are considered as family. Therefore, the majority of these women were alone. In fact, 31 of the interviewed women $(17.2 \%)$ reported being socially isolated and only 75 women (41.7\%) said they were in communication with their family.

Regarding the second block, which analyzed the circumstances of the trip and their reasons for emigration, $21.5 \%$ of women did not report the circumstances of their trip to Spain, $56.9 \%$ reported arriving by plane and $16.0 \%$ by bus. The remaining $5.6 \%$ said they had arrived by car or initially by plane followed by other transports such as train or boat.

In relation to their reasons for emigrating, the majority of the women reported having moved in order to find work and improve their situation (70.7\%), $16.6 \%$ came for family reasons, $4.4 \%$ reported coming for tourism or to complete their education (1.1\%). Only $2.2 \%$ acknowledged having emigrated in order to practice prostitution. A $5 \%$ of cases chose not to state their reasons. 
Differences were noted in the work offer that motivated their emigration according to their origin, $\chi^{2}(26$, $N=180)=55.404, p<.001$. Latin American women, in general, came to Spain without any job offers $(72.7 \%)$ and for family or personal reasons; only $7.1 \%$ of them came knowing that they would engage in prostitution. Most African women also arrived without any job offers $(54.5 \%)$, and only one came with an offer to practice prostitution. European women, in the majority of cases, did not have any job offers $(55.7 \%$ ), but $24.3 \%$ emigrated in order to engage in prostitution. Thus, an offer to practice prostitution prior to their emigration depended on the country of origin of the interviewed women, $\chi^{2}(4$, $N=180)=12.409, p<.05$.

Regarding their first occupation in Spain, 39 women $(21.7 \%)$ did not provide any information. Of the 141 women who answered this question, $59(41.8 \%)$ claimed to have engaged in prostitution, $25(17.7 \%)$ reported not having engaged in any activity, $17(12.1 \%)$ reported working as waitresses (this concept could include women who worked in strip clubs), and 40 women (28.4\%) engaged in other activities of the services sector.

The difference between those who came with an offer to practice prostitution and those who engaged in prostitution upon arrival indicates that at least $22.0 \%$ were deceived through their work offer. This percentage increases to $36.9 \%$ if those that claim to have dedicated themselves to waitressing or strip club workers are taken into consideration. These figures could be even greater if it is considered that many of the 39 women who did not report their occupation when they arrived to Spain could also have engaged in prostitution.

In the third group, which analyzes living and working conditions, 15 of the interviewed women (8.3\%) reported living alone, $139(77.2 \%)$ did not report who they were living with, $72(40.0 \%)$ said they slept in a place different from the strip club, and only $60(33.3 \%)$ women report sleeping at the strip club.

When asked if they can change their address freely, only seven women (3.9\%) answered affirmatively. Although 164 women $(91.1 \%)$ reported having freedom of movement, $30(16.67 \%)$ said they had to be accompanied if they wanted to leave, $148(82.22 \%)$ did not answer and only two women $(1.1 \%)$ stated that they could leave whenever they wanted.

The fact of arriving in Spain with an offer to engage in prostitution does not involve a greater time dedicated to prostitution and a longer stay at the current club, in comparison to women who did not know what they were going to do before they emigrated to Spain, $F(2,61)=2.233$, $p=$ n.s., $\eta^{2}=.076$ and $\mathrm{F} F(2,61)=2.569, p=$ n.s., $\eta^{2}=.087$, respectively. A total of 98 women $(54.4 \%)$ reported engaging in prostitution while doing other jobs.

Wanting to stop practicing prostitution depended on the women's origin, $\chi^{2}(4, N=180)=19.181, p<.001$. A $40.0 \%$ of Europeans, $18.21 \%$ of African women and $8.1 \%$ of Latin American women did not report wanting to stop.

Finally, with regard to the analysis of the intuitive perception of the testimony credibility, the interviewers were asked to issue an intuitive judgment of credibility based on the general analysis of the interview and the context surrounding it, answering two questions: "Was the woman's story believable/unbelievable?" and "Is the woman being interviewed a victim of human trafficking?" The results showed that in $64.4 \%$ of the cases, the testimony given was credible, whereas in the remaining $35.6 \%$ of cases, it was considered to be not credible and therefore, these women could be victims of human trafficking, sexual exploitation or in a situation of high vulnerability. Based on the testimonies heard and what was seen throughout the police procedure, the interviewers indicated that $45.5 \%$ of these prostitutes could have been victims of coercion.

Moreover, a relationship of dependence between the testimony's credibility estimation and the possibility of being a victim of human trafficking was found, $\chi^{2}(1$, $N=180)=89.346, p<.001$. A $94.4 \%$ of women whose testimony was believed to be credible were not considered to be victims of human trafficking; $72.8 \%$ of those estimated as non-credible were considered to be victims of human trafficking.

The analysis of the data showed that the possibility of the interviewees being victims of human trafficking, according to interviewers, depended on their social isolation, 
$\chi^{2}(2, N=180)=5.898, p=.05$, fear or concern shown throughout the interview, $\chi^{2}(2, N=180)=7.501, p<.05$, their lack of collaboration, $\chi^{2}(2, N=180)=10.233$, $p<.01$, their avoidance of certain relevant issues, $\chi^{2}(2$, $N=180)=14.096, p=.001$, being susceptible, $\chi^{2}(2$, $N=180)=1.021, p<.001$, their report of not being hit, $\chi^{2}(2, N=180)=4.284, p<.05$, not suffering any physical abuse, $\chi^{2}(2, N=180)=4.284, p<.05$, or sexual abuse, $\chi^{2}(2, N=180)=4.284, p<.05$, making use of or disposing of their own money, $\chi^{2}(1, N=180)=19.733$, $p<.001$, looking for another job, $\chi^{2}(2, N=180)=8.035$, $p<.05$, and being free to stop prostituting themselves, $\chi^{2}(2, N=180)=11.579, p<.01$.

The main limitation of this study is the interviewers' suspicion in relation to the high percentage of interviewed women who did not provide a credible testimony by systematically denying or not answering questions that would demonstrate abuse or trafficking. 